\title{
The Dirichlet problem with sublinear nonlinearities
}

\author{
Aleksandra OrPel (Łódź)
}

\begin{abstract}
We investigate the existence of solutions of the Dirichlet problem for the differential inclusion $0 \in \Delta x(y)+\partial_{x} G(y, x(y))$ for a.e. $y \in \Omega$, which is a generalized Euler-Lagrange equation for the functional $J(x)=\int_{\Omega}\left\{\frac{1}{2}|\nabla x(y)|^{2}-G(y, x(y))\right\} d y$. We develop a duality theory and formulate the variational principle for this problem. As a consequence of duality, we derive the variational principle for minimizing sequences of $J$. We consider the case when $G$ is subquadratic at infinity.
\end{abstract}

1. Introduction. We study the following differential inclusion:

$$
0 \in \Delta x(y)+\partial_{x} G(y, x(y)) \quad \text { for a.e. } y \in \Omega,
$$

where $\Omega \subset \mathbb{R}^{n}, \Delta$ is the Laplace operator and $\partial_{x} G(y, x)$ denotes the subdifferential of the function $\mathbb{R} \ni x \mapsto G(y, x), y \in \Omega$. We will prove the existence of a solution for (1.1) in the subspace $W_{0}^{1,2}(\Omega, \mathbb{R})$ consisting of all functions in $W^{1,2}(\Omega, \mathbb{R})$ satisfying the boundary condition $\left.x\right|_{\partial \Omega}=0$. To this end we use variational methods and we consider the above inclusion as a generalized Euler-Lagrange equation for the functional $J: W_{0}^{1,2}(\Omega, \mathbb{R}) \rightarrow \mathbb{R}$ given by

$$
J(x)=\int_{\Omega}\left\{\frac{1}{2}|\nabla x(y)|^{2}-G(y, x(y))\right\} d y .
$$

Our approach covers the case when the map $G$ is subquadratic at infinity.

Hypothesis $(\mathrm{H})$. Let $\Omega$ be a bounded domain in $\mathbb{R}^{n}$ having a locally Lipschitz boundary. Assume that the function $G: \Omega \times \mathbb{R} \rightarrow \mathbb{R}$ is measurable with respect to the first variable, convex with respect to the second and

$$
\frac{1}{p} a|x|^{p}+k(y) \leq G(y, x) \leq \frac{1}{r} b|x|^{r}+l(y)
$$

for a.e. $y \in \Omega$ and $x \in \mathbb{R}$, where $a, b \in(0, \infty), k, l \in L^{1}(\Omega, \mathbb{R}), 1<p<r \leq 2$.

2000 Mathematics Subject Classification: Primary 58E30; Secondary 35J20, 35J25.

Key words and phrases: Dirichlet problem, duality, variational principle, EulerLagrange equation. 
This paper is organized as follows: first we show the existence of a critical point $\bar{x} \in W_{0}^{1,2}(\Omega, \mathbb{R})$ for $(1.2)$. In the next section we develop a duality theory describing a relation between $J$ and a dual object, the functional $J_{D}$, which will be introduced below. We also use the concept of the Fenchel conjugate. The duality principle proved in Section 2 will be used to obtain the existence of a minimizer $\bar{p}$ for $J_{D}$ and two interesting equalities which $\bar{x}$ and $\bar{p}$ must satisfy. As a consequence, we shall prove that $\bar{x}$ is a solution of the elliptic partial differential inclusion (1.1) with the boundary condition $\left.x\right|_{\partial \Omega}=0$.

Several authors have dealt with this and similar problems (see e.g. [8], $[9],[7],[15])$. However the present paper takes a new viewpoint. It is worth noting that we do not impose any regularity on $G$ or $G^{*}$, where $G^{*}(y, v)=$ $\sup _{x \in \mathbb{R}}[\langle v, x\rangle-G(y, x)]$ for $y \in \Omega$ and $v \in \mathbb{R}$. That is why our method is different from what was used in previous papers. The idea of the method presented here is similar to that of [11] and [12], where the Dirichlet problem for ordinary differential equations is studied.

If we assume stronger conditions concerning the smoothness of $G$, its derivative $G_{x}\left(G_{x}(y, x):=\frac{d}{d x} G(y, x)\right)$ and some additional estimates on $G_{x}$, we can obtain the existence result for (1.1) from the existence of a minimum of energy. In [9], where the one-dimensional case has been discussed, $G$ is continuously differentiable with respect to the second variable, moreover $G$ and $G_{x}$ satisfy some special growth conditions. These assumptions are associated with the existence of the Gateaux differential for the given functional.

In the general case described by hypothesis $(H)$, inclusion (1.1) cannot be deduced from the existence of a minimum of $J$. In order to obtain the existence of a solution for (1.1) we develop a duality theory. As we see in Section 5, the duality principle enables a numerical characterization of minimizing sequences and approximation of the infimum of (1.2). Moreover we are able to establish an approximate solution for (1.1).

For the reader's convenience, we repeat relevant material from [2] and [4] without proofs.

TheOREM 1.1. Let $W: \mathbf{Y} \rightarrow \mathbb{R} \cup\{+\infty\}$ be a proper, convex and lower semicontinuous function, and $A \in L(\mathbf{X}, \mathbf{Y})$, where $\mathbf{X}, \mathbf{Y}$ are Hilbert spaces. If

$$
0 \in \operatorname{Int}(\operatorname{Im} A-\operatorname{dom} W),
$$

then for every $p \in A^{*}\left(\operatorname{dom} W^{*}\right)$, there exists $\bar{q} \in \mathbf{Y}^{*}$ satisfying

$$
A^{*} \bar{q}=p, \quad(W A)^{*}(p)=W^{*}(\bar{q})=\inf _{A^{*} q=p} W^{*}(q),
$$

where $A^{*}$ and $W^{*}$ denote the Fenchel conjugates of $A$ and $W$, respectively. 
Theorem 1.2. Assume that $F_{0}: \Omega \times \mathbb{R}^{n+r} \rightarrow \mathbb{R}$ satisfies the Carathéodory condition, $F_{0}$ is convex with respect to the last variable and there exist $\phi \in L^{1}\left(\Omega, \mathbb{R}_{+} \cup\{0\}\right)$ and $C \geq 0$ such that for all $(u, w) \in \mathbb{R}^{n+r}$ and a.e. $x \in \Omega$,

$$
F_{0}(x, u, w) \geq-\phi(x)-C|w| .
$$

If $\zeta, \zeta_{k}, u, u_{k}, k=1,2, \ldots$, are measurable functions such that $\zeta, \zeta_{k} \in$ $\left(L^{1}(\Omega)\right)^{r}, k=1,2, \ldots, u_{k} \rightarrow u$ in measure on $\Omega$ and $\zeta_{k} \rightarrow \zeta$ weakly in $\left(L^{1}(\Omega)\right)^{r}$, then

$$
\int_{\Omega} F_{0}(x, u(x), \zeta(x)) d x \leq \liminf _{k \rightarrow \infty} \int_{\Omega} F_{0}\left(x, u_{k}(x), \zeta_{k}(x)\right) d t .
$$

2. The existence of a minimum for $J$. Now we prove the existence of a minimum for the functional $J$. To this end we have to make some additional assumptions concerning the set $\Omega$ :

Hypothesis (H1). Let $\Omega \subset B(z, R)$, where $B(z, R)$ is the ball centered at $z$ with radius $R$. In the case $r=2$ we assume that $\sqrt{p^{2} / b}>R$.

Throughout the paper, unless otherwise stated, we shall assume hypotheses $(\mathrm{H})$ and $(\mathrm{H} 1)$.

Theorem 2.1. There exists $\bar{x} \in W_{0}^{1,2}(\Omega, \mathbb{R})$ such that

$$
-\infty<J(\bar{x})=\inf _{x \in W_{0}^{1,2}(\Omega, \mathbb{R})} J(x)<\infty .
$$

Proof. By the assumptions on $G$, we obtain

$$
\begin{aligned}
J(x) & =\int_{\Omega}\left\{\frac{1}{2}|\nabla x(y)|^{2}-G(y, x(y))\right\} d y \\
& \geq \int_{\Omega}\left\{-\frac{1}{r} b|x(y)|^{r}+\frac{1}{2}|\nabla x(y)|^{2}-l(y)\right\} d y \\
& \geq \int_{\Omega}\left\{-\frac{1}{r} b|x(y)|^{r}+\frac{p^{2}}{2 R^{2}}|x(y)|^{2}-l(y)\right\} d y,
\end{aligned}
$$

where the last relation is a consequence of the Poincaré inequality ([10]).

When $r<2$, this chain of relations gives the lower boundedness of $J$ on $W^{1,2}(\Omega, \mathbb{R})$ and on $W_{0}^{1,2}(\Omega, \mathbb{R})$. Using hypothesis $(\mathrm{H} 1)$, we get the same conclusion in the case $r=2$. The above condition and (H1) imply that there is a minimizing sequence $\left\{x_{n}\right\}_{n \in \mathbb{N}} \subset W_{0}^{1,2}(\Omega, \mathbb{R})$ for $J$, which is bounded in the $\|\cdot\|_{W^{1,2}(\Omega, \mathbb{R})}$ norm. Hence we can choose a subsequence $\left\{x_{n_{k}}\right\}_{k \in \mathbb{N}}$ weakly convergent to $\bar{x} \in W^{1,2}(\Omega, \mathbb{R})$. Since $W_{0}^{1,2}(\Omega, \mathbb{R})$ is weakly closed in $W^{1,2}(\Omega, \mathbb{R})$, we have $\bar{x} \in W_{0}^{1,2}(\Omega, \mathbb{R})$. Applying Theorem 1.2 we obtain

$$
J(\bar{x}) \leq \liminf _{k \rightarrow \infty} J\left(x_{n_{k}}\right)
$$


and further, from the definition of $\left\{x_{n}\right\}_{n \in \mathbb{N}}$,

$$
J(\bar{x})=\inf _{x \in W_{0}^{1,2}(\Omega, \mathbb{R})} J(x) .
$$

3. Duality principles. Because hypothesis $(\mathrm{H})$ is not sufficient to deduce the existence of a solution for (1.1) from Theorem 2.1, we develop a duality theory. Using hypothesis $(\mathrm{H})$ we can see that the functionals introduced below are well defined. Set

$$
\begin{aligned}
& C=\left\{p \in L^{2}\left(\Omega, \mathbb{R}^{n}\right) ; \operatorname{div} p(y)=0 \text { for a.e. } y \in \Omega\right\}, \\
& B=\left\{p \in L^{2}\left(\Omega, \mathbb{R}^{n}\right) ; \operatorname{div} p \in L^{2}(\Omega, \mathbb{R})\right\} .
\end{aligned}
$$

As usual, $B+C=\{b+c ; b \in B$ and $c \in C\}$.

We will prove a duality principle describing the relations between $J$ and the dual functional $J_{D}: B+C \rightarrow \mathbb{R}$ given by

$$
J_{D}(p+v)=\int_{\Omega}\left\{-\frac{1}{2}|p(y)+v(y)|^{2}+G^{*}(y,-\operatorname{div} p(y))\right\} d y .
$$

Define the perturbation $J_{x}: L^{2}(\Omega, \mathbb{R}) \rightarrow \mathbb{R}$ of $J$ by

$$
J_{x}(g)=\int_{\Omega}\left\{-\frac{1}{2}|\nabla x(y)|^{2}+G(y, x(y)+g(y))\right\} d y .
$$

Also define the functional $J_{x}^{\#}: B \rightarrow \mathbb{R}$ for $x \in W_{0}^{1,2}(\Omega, \mathbb{R})$ by

$$
J_{x}^{\#}(p)=\sup _{g \in L^{2}(\Omega, \mathbb{R})} \int_{\Omega}\left\{\langle\operatorname{div} p(y), g(y)\rangle-G(y, x(y)+g(y))+\frac{1}{2}|\nabla x(y)|^{2}\right\} d y \text {. }
$$

After some calculations, we can give an equivalent and simpler form of $J_{x}^{\# \text { : }}$

$$
J_{x}^{\#}(p)=\int_{\Omega}\left\{\langle-\operatorname{div} p(y), x(y)\rangle+G^{*}(y, \operatorname{div} p(y))+\frac{1}{2}|\nabla x(y)|^{2}\right\} d y .
$$

Let

$$
A=\left\{\nabla x \in L^{2}\left(\Omega, \mathbb{R}^{n}\right) ; x \in W_{0}^{1,2}(\Omega, \mathbb{R})\right\} .
$$

Using (3.1), (3.3) and Theorem 1.1, we obtain

$$
\begin{aligned}
\sup _{x \in W_{0}^{1,2}(\Omega, \mathbb{R})}\left(-J_{x}^{\#}(-p)\right) \\
=\sup _{\nabla x \in A} \int_{\Omega}\left\{\langle p(y), \nabla x(y)\rangle-\frac{1}{2}|\nabla x(y)|^{2}-G^{*}(y,-\operatorname{div} p(y))\right\} d y \\
=\inf _{v \in C} \int_{\Omega}\left\{\frac{1}{2}|p(y)+v(y)|^{2}-G^{*}(y,-\operatorname{div} p(y))\right\} d y \\
=\inf _{v \in C}\left(-J_{D}(p+v)\right) .
\end{aligned}
$$


We now introduce the functional $J_{x}^{\# \#}: L^{2}(\Omega, \mathbb{R}) \rightarrow \mathbb{R}$ for $x \in W_{0}^{1,2}(\Omega, \mathbb{R})$ by

$$
\begin{aligned}
& J_{x}^{\# \#}(g) \\
& =\sup _{h \in D} \int_{\Omega}\left\{\langle g(y), h(y)\rangle+\langle x(y), h(y)\rangle-\frac{1}{2}|\nabla x(y)|^{2}-G^{*}(y, h(y))\right\} d y
\end{aligned}
$$

where

$$
D=\left\{\operatorname{div} p \in L^{2}(\Omega, \mathbb{R}) ; p \in B\right\} .
$$

Then $J_{x}^{\# \#}(0)=-J(x)$ for all $x \in W_{0}^{1,2}(\Omega, \mathbb{R})$. Indeed, by the definitions of $J_{x}^{\# \#}$ and $D$, we have

$$
\begin{aligned}
J_{x}^{\# \#}(0) & =\sup _{h \in L^{2}(\Omega, \mathbb{R})} \int_{\Omega}\left\{\langle x(y), h(y)\rangle-\frac{1}{2}|\nabla x(y)|^{2}-G^{*}(y, h(y))\right\} d y \\
& =\int_{\Omega}\left\{-\frac{1}{2}|\nabla x(y)|^{2}+G(y, x(y))\right\} d y=-J(x) .
\end{aligned}
$$

Now we can prove the following

THEOREM 3.1.

$$
\inf _{x \in W_{0}^{1,2}(\Omega, \mathbb{R})} J(x)=\inf _{\operatorname{div} p \in D} \sup _{v \in C} J_{D}(p+v) .
$$

Proof. From (3.5), (3.4) and the definitions of $C$ and $D$, we deduce

$$
\begin{aligned}
\sup _{x \in W_{0}^{1,2}(\Omega, \mathbb{R})} J_{x}^{\# \#}(0)= & \sup _{x \in W_{0}^{1,2}(\Omega, \mathbb{R}) \operatorname{div} p \in D} \sup _{\Omega}\{\langle x(y),-\operatorname{div} p(y)\rangle \\
& \left.-G^{*}(y,-\operatorname{div} p(y))-\frac{1}{2}|\nabla x(y)|^{2}\right\} d y \\
= & \sup _{\operatorname{div} p \in D} \sup _{x \in W_{0}^{1,2}(\Omega, \mathbb{R})}\left(-J_{x}^{\#}(-p)\right)=\sup _{\operatorname{div} p \in D} \inf \left(-J_{D}(p+v)\right) \\
= & -\inf _{\operatorname{div} p \in D} \sup _{v \in C} J_{D}(p+v) .
\end{aligned}
$$

The above and the equality $J_{x}^{\# \#}(0)=-J(x)$ imply

$$
\inf _{x \in W_{0}^{1,2}(\Omega, \mathbb{R})} J(x)=\inf _{\operatorname{div} p \in D} \sup _{v \in C} J_{D}(p+v) .
$$

4. Variational principles. We now formulate and prove the variational principle for minimizers. From these results we will obtain the existence of a solution of (1.1).

THEOREM 4.1. Let $\bar{x} \in W_{0}^{1,2}(\Omega, \mathbb{R})$ and $J(\bar{x})=\inf _{x \in W_{0}^{1,2}(\Omega, \mathbb{R})} J(x)$. There exists $\bar{p}=\widetilde{p}+\bar{v} \in B+C$ such that $-\operatorname{div} \bar{p} \in \partial J_{\bar{x}}(0)$ (where $\partial J_{\bar{x}}(0)$ 
denotes the subdifferential of $L^{2}(\Omega, \mathbb{R}) \ni g \mapsto J_{\bar{x}}(g)$ at 0$)$ and $\bar{p}$ satisfies

$$
J_{D}(\bar{p})=\inf _{\operatorname{div} p \in D} \sup _{v \in C} J_{D}(p+v)
$$

Moreover,

$$
\begin{aligned}
& J_{\bar{x}}^{\#}(-\bar{p})+J_{\bar{x}}(0)=0 \\
& J_{\bar{x}}^{\#}(-\bar{p})-J_{D}(\bar{p})=0
\end{aligned}
$$

Proof. From Theorem 2.1, we deduce the existence of an $\bar{x} \in W_{0}^{1,2}(\Omega, \mathbb{R})$ such that $J(\bar{x})=\inf _{x \in W_{0}^{1,2}(\Omega, \mathbb{R})} J(x)$. According to $(\mathrm{H})$, the functional

$$
L^{2}(\Omega, \mathbb{R}) \ni z \mapsto M(z)=-\int_{\Omega}\left\{\langle\bar{x}(y),-z(y)\rangle-G^{*}(y,-z(y))\right\} d y
$$

is convex, lower semicontinuous and coercive. Moreover $L^{2}(\Omega, \mathbb{R})$ is a reflexive space. Therefore the map $M$ attains its infimum at some $\bar{z} \in L^{2}(\Omega, \mathbb{R})$. On the other hand,

$$
\begin{aligned}
\sup _{z \in L^{2}(\Omega, \mathbb{R})}(-M(z)) & =\sup _{z \in L^{2}(\Omega, \mathbb{R})} \int_{\Omega}\left\{\langle\bar{x}(y),-z(y)\rangle-G^{*}(y,-z(y))\right\} d y \\
& =\int_{\Omega} G(y, \bar{x}(y)) d y .
\end{aligned}
$$

Combining these results we deduce

$$
\begin{aligned}
& \int_{\Omega}\left\{G(y, \bar{x}(y))-\frac{1}{2}|\nabla \bar{x}(y)|^{2}\right\} d y \\
& \quad+\int_{\Omega}\left\{\langle\bar{x}(y), \bar{z}(y)\rangle+G^{*}(y,-\bar{z}(y))+\frac{1}{2}|\nabla \bar{x}(y)|^{2}\right\} d y=0 .
\end{aligned}
$$

Let $\widetilde{p} \in B$ and $\operatorname{div} \widetilde{p}=\bar{z}$ (we write for brevity

$$
\widetilde{p}(y)=(\int_{a_{1}}^{y_{1}} \bar{z}\left(t, y_{2}, \ldots, y_{n}\right) d t, \underbrace{0, \ldots, 0}_{n-1}),
$$

where $y=\left(y_{1}, \ldots, y_{n}\right)$, and $a=\left(a_{1}, \ldots, a_{n}\right)$ is a certain element of $\left.\Omega\right)$. Writing $\widetilde{p}_{v}=\widetilde{p}+v$, where $v \in C$, we get $\operatorname{div} \widetilde{p}_{v}=\operatorname{div} \widetilde{p}=\bar{z}$. Applying (4.4) we have

$$
J_{\bar{x}}^{\#}\left(-\widetilde{p}_{v}\right)+J_{\bar{x}}(0)=0
$$

We also note that

$$
J_{\bar{x}}^{*}\left(-\operatorname{div} \widetilde{p}_{v}\right)=J_{\bar{x}}^{\#}\left(-\widetilde{p}_{v}\right),
$$

where $J_{\bar{x}}^{*}$ is the polar of $J_{\bar{x}}$. Indeed, 


$$
\begin{aligned}
& J_{\bar{x}}^{*}\left(-\operatorname{div} \widetilde{p}_{v}\right) \\
& =\sup _{g \in L^{2}(\Omega, \mathbb{R})} \int_{\Omega}\left\{\left\langle-\operatorname{div} \widetilde{p}_{v}(y), g(y)\right\rangle+\frac{1}{2}|\nabla \bar{x}(y)|^{2}-G(y, \bar{x}(y)+g(y))\right\} d y \\
& \quad=\int_{\Omega}\left\{\frac{1}{2}|\nabla \bar{x}(y)|^{2}+\left\langle\operatorname{div} \widetilde{p}_{v}(y), \bar{x}(y)\right\rangle+G^{*}\left(y,-\operatorname{div} \widetilde{p}_{v}(y)\right)\right\} d y=J_{\bar{x}}^{\#}\left(-\widetilde{p}_{v}\right) .
\end{aligned}
$$

Substituting (4.6) into (4.5) gives $-\operatorname{div} \widetilde{p}_{v} \in \partial J_{\bar{x}}(0)$ for every $v \in C$.

Consider the map

$$
C \ni v \mapsto h(v)=-\int_{\Omega}\left\{-\frac{1}{2}|\widetilde{p}(y)+v(y)|^{2}+G^{*}(y,-\operatorname{div} \widetilde{p}(y))\right\} d y .
$$

By hypothesis $(\mathrm{H})$ this functional is convex, lower semicontinuous and coercive. Thus, by the reflexivity of the space $C, h$ attains its minimum at $\bar{v} \in C$. Using the fact that $J_{\bar{x}}(0)=-J(\bar{x}),(4.5)$ and (3.4) we deduce that

$$
-J(\bar{x})=-J_{\bar{x}}^{\#}\left(-\widetilde{p}_{v}\right) \leq \sup _{x \in W_{0}^{1,2}(\Omega, \mathbb{R})}\left(-J_{x}^{\#}\left(-\widetilde{p}_{v}\right)\right)=-\sup _{v \in C} J_{D}(\widetilde{p}+v)=-J_{D}(\bar{p}),
$$

where $\bar{p}=\widetilde{p}+\bar{v}$. Thus Theorem 3.1 implies (4.1).

Combining the equality $\operatorname{div} \bar{p}=\operatorname{div} \widetilde{p}$ and (4.5) then gives (4.2); (4.3) is a simple consequence of $(4.2)$ and $J_{D}(\bar{p})=J(\bar{x})=-J_{\bar{x}}(0)$.

THEOREM 4.2. There exists a solution $\bar{x} \in W_{0}^{1,2}(\Omega, \mathbb{R})$ of the inclusion

$$
0 \in \Delta x(y)+\partial_{x} G(y, x(y)) \quad \text { for a.e. } y \in \Omega \text {. }
$$

Proof. In view of Theorem 2.1 we deduce the existence of a minimizer $\bar{x}$ of $J: W_{0}^{1,2}(\Omega, \mathbb{R}) \rightarrow \mathbb{R}$. Using Theorem 4.1 we can find $\bar{p}=\widetilde{p}+\bar{v} \in B+C$ satisfying (4.1). Applying (4.2) yields

$$
G(y, \bar{x}(y))+G^{*}(y,-\operatorname{div} \bar{p}(y))-\langle\bar{x}(y),-\operatorname{div} \bar{p}(y)\rangle=0
$$

for a.e. $y \in \Omega$. Hence, by the properties of the subdifferential, we obtain

$$
-\operatorname{div} \bar{p}(y) \in \partial_{x} G(y, \bar{x}(y))
$$

for a.e. $y \in \Omega$. On the other hand, (4.3) gives $\nabla \bar{x}(y)=\bar{p}(y)$ and further

$$
\Delta \bar{x}(y)=\operatorname{div} \bar{p}(y)
$$

for a.e. $y \in \Omega$. Combining these relations completes the proof.

REMARK 4.1. When $G$ is differentiable in the second variable and $G_{x}(y, x)$ denotes the differential of $\mathbb{R} \ni x \mapsto G(y, x), y \in \Omega$, Theorem 4.2 shows that there exists a solution $\bar{x} \in W^{1,2}(\Omega, \mathbb{R})$ of the Dirichlet problem for the elliptic partial differential equation

$$
\Delta x(y)+G_{x}(y, x(y))=0
$$

for a.e. $y \in \Omega$. 
5. Minimizing sequences. In this section we present another application of the duality principle. We prove a theorem for minimizing sequences of $J$ analogous to Theorem 4.1. Due to these results we obtain a numerical approximation of the minimum of $J$.

THEOREM 5.1. Let $\left\{x_{n}\right\}_{n \in \mathbb{N}} \subset W_{0}^{1,2}(\Omega, \mathbb{R})$ be a minimizing sequence for $J$ on $W_{0}^{1,2}(\Omega, \mathbb{R})$. Then for all $n \in \mathbb{N}$ there exists $p_{n}=p_{n}^{0}+v_{n} \in B+C$ such that

$$
\begin{aligned}
& -\operatorname{div} p_{n} \in \partial J_{x_{n}}(0), \\
& \sup _{v \in C} J_{D}\left(p_{n}^{0}+v\right)=J_{D}\left(p_{n}^{0}+v_{n}\right), \\
& \inf _{n \in \mathbb{N}} J_{D}\left(p_{n}\right)=\inf _{\operatorname{div} p \in D} \sup _{v \in C} J_{D}(p+v) .
\end{aligned}
$$

Moreover for all $n \in \mathbb{N}$,

$$
J_{x_{n}}(0)+J_{x_{n}}^{\#}\left(-p_{n}\right)=0
$$

and for given $\varepsilon>0$, there exists $n_{0} \in \mathbb{N}$ such that for $n>n_{0}$,

$$
\begin{aligned}
J_{x_{n}}^{\#}\left(-p_{n}\right)-J_{D}\left(p_{n}\right) & \leq \varepsilon, \\
\left|J_{D}\left(p_{n}\right)-J\left(x_{n}\right)\right| & \leq \varepsilon .
\end{aligned}
$$

Proof. By hypothesis (H),

$$
\inf _{x \in W_{0}^{1,2}(\Omega, \mathbb{R})} J(x)=a>-\infty .
$$

From the definition of $a$ we get

$$
a=\inf _{x \in W_{0}^{1,2}(\Omega, \mathbb{R})} J(x)=\inf _{n \in \mathbb{N}} J\left(x_{n}\right) .
$$

This implies that for given $\varepsilon>0$, there exists $n_{0} \in \mathbb{N}$ such that $\varepsilon>J\left(x_{n}\right)-a$ for $n>n_{0}$. From now on, the proof is similar to that of Theorem 4.1. Analogously we obtain for all $n \in \mathbb{N}$ the existence of $p_{n}^{0} \in B$ such that (5.2) holds. Combining (5.2) and the equality $J_{x_{n}}^{*}\left(-\operatorname{div} p_{n}\right)=J_{x_{n}}^{\#}\left(-p_{n}\right)$, we conclude that $-\operatorname{div} p_{n} \in \partial J_{x_{n}}(0)$ for all $n \in \mathbb{N}$. Now using the definition of the perturbation $J_{x}$ and the above results, we may infer that for all $n \in \mathbb{N}$,

$$
J\left(x_{n}\right)-J_{x_{n}}^{\#}\left(-p_{n}\right)=0 .
$$

On the other hand, $\varepsilon+a>J\left(x_{n}\right)$ for $n>n_{0}$. Thus we obtain

$$
-a-\varepsilon<-J_{x_{n}}^{\#}\left(-p_{n}^{0}\right) \leq \sup _{x \in W_{0}^{1,2}(\Omega, \mathbb{R})}\left(-J_{x}^{\#}\left(-p_{n}^{0}\right)\right)=-\sup _{v \in C} J_{D}\left(p_{n}^{0}+v\right),
$$

where the last equality follows from (3.4).

Using the ideas of the proof of Theorem 4.1, we deduce that for each $n \in \mathbb{N}$, there exists $v_{n} \in C$ such that

$$
\sup _{v \in C} J_{D}\left(p_{n}^{0}+v\right)=J_{D}\left(p_{n}^{0}+v_{n}\right) .
$$


Taking $p_{n}=p_{n}^{0}+v_{n}, n \in \mathbb{N}$, we also deduce that for given $\varepsilon>0$, there exists $n_{0} \in \mathbb{N}$ such that $J_{D}\left(p_{n}\right)<a+\varepsilon$ for $n>n_{0}$. This means that

$$
a=\inf _{n \in \mathbb{N}} J_{D}\left(p_{n}\right) .
$$

Thus, by Theorem 3.1, we have

$$
\inf _{n \in \mathbb{N}} J_{D}\left(p_{n}\right)=\inf _{p \in W_{0}^{1,2}\left(\Omega, \mathbb{R}^{n}\right)} J_{D}(p) .
$$

Finally, we recall that $J_{x_{n}}^{\#}\left(-p_{n}\right) \leq a+\varepsilon$ for $n>n_{0}$. From this fact and the previous assertion, we get (5.3). The last inequality is a consequence of the definition of $a$ and the equality (5.7).

6. An application. Now we give an application of this theory for a class of PDE of elliptic type.

EXAMPLE 6.1. Let $m \in \mathbb{N}$ and let $\Omega \subset \mathbb{R}^{n}$ be a bounded, locally Lipschitz domain. Consider the function $G$ defined as follows:

$$
G(y, x)=c(y)|x|^{(m+1) / m}-x f(y),
$$

where $f \in L^{2}(\Omega, \mathbb{R}), c \in L^{\infty}(\Omega, \mathbb{R})$ and there exists $a \in(0, \infty)$ such that $a \leq c(y)$ for a.e. $y \in \Omega$. Then there exists a solution $x_{0} \in W_{0}^{1,2}(\Omega, \mathbb{R})$ of the Dirichlet problem for the following PDE:

$$
\Delta x(y)+\operatorname{sgn}(x(y)) \frac{m+1}{m} c(y)|x(y)|^{1 / m}=f(y) .
$$

Proof. The function $G$ satisfies all the assumptions of hypothesis $(\mathrm{H})$. Applying Theorem 4.2 (or Remark 4.1) we obtain the existence of a solution of the Dirichlet problem for a particular case of inclusion (1.1):

$$
\Delta x(y)+\operatorname{sgn}(x(y)) \frac{m+1}{m} c(y)|x(y)|^{1 / m}=f(y)
$$

for a.e. $y \in \Omega$.

\section{References}

[1] R. A. Adams, Sobolev Spaces, Academic Press, New York, 1975.

[2] J. P. Aubin and I. Ekeland, Applied Nonlinear Analysis, Wiley, New York 1984.

[3] I. Ekeland and R. Temam, Convex Analysis and Variational Problems, NorthHolland, Amsterdam, 1976.

[4] L. Cesari, Optimization-Theory and Applications, Springer, New York, 1983.

[5] F. H. Clarke, Optimization and Nonsmooth Analysis, Wiley, New York, 1983.

[6] D. Gilbarg and N. S. Trudinger, Elliptic Partial Differential Equations of Second Order, Springer, 1983.

[7] I. Kuzin and S. Pokhozhaev, Entire Solutions of Semilinear Elliptic Equations, Progr. Nonlinear Differential Equations Appl. 33, Birkhäuser, Basel, 1997.

[8] J. Mawhin, Problèmes de Dirichlet variationnels non linéaires, Presses Univ. Montréal, 1987. 
[9] J. Mawhin and M. Willem, Critical Points Theory and Hamiltonian Systems, Springer, New York, 1989.

[10] C. B. Morrey, Multiple Integrals in the Calculus of Variations, Springer, Berlin, 1966.

[11] A. Nowakowski, Variational Methods for Nonlinear Dirichlet Problems (Chapter 6), Wydawnictwa Naukowo-Techniczne, Warszawa, 1994 (in Polish).

[12] - Duality and classical problem of the calculus of variations without convexity assumptions, Optimization 21 (1990), 575-584.

[13] A. Orpel, The existence of the critical points for the action functional without convexity assumption, ibid. 44 (1998), 105-122.

[14] R. T. Rockafellar, Conjugate convex functions in optimal control and the calculus of variations, J. Math. Anal. Appl. 32 (1970), 174-222.

[15] M. Willem, Minimax Theorems, Progr. Nonlinear Differential Equations Appl. 24, Birkhäuser, Basel, 1996.

Faculty of Mathematics

University of Łódź

Banacha 22

90-238 Łódź, Poland

E-mail: orpela@math.uni.lodz.pl

Received on 15.11.2000;

revised version on 19.4.2001 